\title{
A Bernstein theorem for complete spacelike constant mean curvature hypersurfaces in Minkowski space
}

\author{
Huai-Dong Cao Ying Shen Shunhui Zhu
}

\begin{abstract}
We obtain a gradient estimate for the Gauss maps from complete spacelike constant mean curvature hypersurfaces in Minkowski space into the hyperbolic space. As applications, we prove a Bernstein theorem which says that if the image of the Gauss map is bounded from one side, then the spacelike constant mean curvature hypersurface must be linear. This result extends the previous theorems obtained by B. Palmer [Pa] and Y.L. Xin Xin] where they assume that the image of the Gauss map is bounded. We also proved a Bernstein theorem for spacelike complete surfaces with parallel mean curvature vector in four-dimensional spaces.
\end{abstract}

\section{Introduction}

The classical Bernstein theorem [B] states that the only entire solution of the minimal hypersurface equation in Euclidean space $\mathbb{R}^{n+1}$

$$
\sum_{i=1}^{n} \frac{\partial}{\partial x_{i}}\left(\frac{\frac{\partial u}{\partial x_{i}}}{\sqrt{1+|\nabla u|^{2}}}\right)=0
$$

\footnotetext{
${ }^{1} 1991$ Mathematics Subject Classification. Primary 53C21, 53C42.

${ }^{2}$ Key words and phrases. Spacelike hypersurfaces, constant mean curvature, harmonic maps, gradient estimates.

${ }^{3}$ The first author's research was supported in part by NSF grant \#DMS-9504925. The third author's research was supported in part by NSF grant \#DMS-9404263
} 
is linear for $n=2$. The higher dimensional version of the Bernstein theorem was proved through the efferts of Federer, Fleming [F], De Giorgi [DG], Almgren [A], Simons [S.]]. They showed that the only entire solution of Eq.(1) is linear for $n \leq 7$ while Bombieri-De Giorgi-Giusti [BDG] showed that there are nontrivial solutions for $n>7$. In 1965, Chern [Ch] proved that the only entire solution of constant mean curvature hypersurface equation in $\mathbb{R}^{m+1}$

$$
\sum_{i=1}^{m} \frac{\partial}{\partial x_{i}}\left(\frac{\frac{\partial u}{\partial x_{i}}}{\sqrt{1+|\nabla u|^{2}}}\right)=m H
$$

is also linear for $2 \leq m \leq 7$. Then he proposed the generalized Bernstein problem by studying the distribution of normal vectors to a complete constant mean curvature hypersurface. A nice result in this direction is a theorem due to Hoffman, Osserman, and Schoen [H-O-S which states that if the normal vectors to a complete constant mean curvature surface in $\mathbb{R}^{3}$ lie in an open hemisphere, then it is a plane.

In 1968, Calabi Ca proposed to study a similar problem in the Minkowski space. He considered the maximal spacelike hypersurface equation

$$
\sum_{i=1}^{m} \frac{\partial}{\partial x_{i}}\left(\frac{\frac{\partial u}{\partial x_{i}}}{\sqrt{1-|\nabla u|^{2}}}\right)=0
$$

in the Lorentz-Minkowski space $\mathbb{R}_{1}^{m+1}$ with coordinate $\left(x_{0}, x_{1}, \cdots, x_{m}\right)$ and metric $d s^{2}=-\left(d x_{0}\right)^{2}+\sum_{i=1}^{m}\left(d x_{i}\right)^{2}$. The Lorentz metric induces a Riemannian metric on the graph of $u$ if $|\nabla u|<1$. In [Ca], Calabi showed that, for $2 \leq m \leq 4$, the graph of any solution to Eq.(2) has to be a hyperplane. Later, Cheng and Yau [C-Y] proved that the same conclusion holds for $m>4$. In fact they proved the parametric version of this theorem: The only maximal spacelike hypersurface which is a closed subset of the Lorentz-Minkowski space (with respect to the Euclidean topology) is a linear hyperplane.

Note that a maximal spacklike hypersurface is a spacelike hypersurface with zero constant mean curvature. In [Tr], Treibergs showed that the constant mean curvature spacelike hypersurface equation

$$
\sum_{i=1}^{m} \frac{\partial}{\partial x_{i}}\left(\frac{\frac{\partial u}{\partial x_{i}}}{\sqrt{1-|\nabla u|^{2}}}\right)=m H
$$


where $|\nabla u|<1$ and $H>0$, has many solutions besides hyperboloids. One important fact in the study of constant mean curvature spacelike hypersurfaces of Minkowski space is that the Gauss map of such a hypersurface $M$ is a harmonic map to hyperbolic space. Let us first recall how the Gauss map is defined. If we let $\nu$ be the timelike unit normal vector field to $M$ in $\mathbb{R}_{1}^{m+1}$. For any point $p \in M,|\nu(p)|^{2}=-1$. By parallel translation to the origin in $\mathbb{R}_{1}^{m+1}$ we can regard $\nu(p)$ as a point in $m$-dimensional hyperbolic space $\mathbb{I H}^{m}(-1)$ which is canonically embedded in $\mathbb{R}_{1}^{m+1}$. The construction gives us a Gauss map $f: M \rightarrow \mathbb{H}^{m}(-1)$. In [Cho-Tr], Choi and Treibergs did an extensive study of the interplay of properties of such a hypersurface $M$ and behavior of its Gauss map. As a result, they constructed various harmonic maps from $\mathbb{R}^{2}$ to $\mathbb{H}^{2}$ and $\mathbb{H}^{2}$ to $\mathbb{H}^{2}$.

Naturally, one can ask under what conditions on the Gauss map a complete spacelike hypersurfaces with constant mean curvature has the Bernstein property. In 1990, Palmer $[\mathrm{Pa}]$ proved that there exists a constant $\tau=\tau(n, H)>0$ such that if the Gauss image of the hypersurface $f(M)$ is contained in a geodesic ball of radius $\tau_{1}<\tau$ in $\mathbb{H}^{m}(-1)$, then $M$ is a hyperplane. Later, Xin Xin1 extended Palmer's theorem by proving that $M$ must be a hyperplane if the image under the Gauss map is bounded.

Our main result in this paper is to find some natural geometric condition and to further extend Palmer and Xin's Bernstein theorem by only assuming that the image under the Gauss map is bounded from one side. The main theorem in Choi and Treibergs' paper [Cho-Tr] states that given an arbitrary closed set in the ideal boundary at infinity of hyperbolic space, there exist a complete entire spacelike constant mean curvature hypersurface whose Gauss map is a diffeomorphism onto the interior of the hyperbolic space convex hull of the set. Therefore, if the closure of the image under the Gauss map intersects the ideal boundary of hyperbolic space at more than one point, Choi-Treibergs' theorem implies that the Bernstein theorem is not true. Therefore, it is natural to conjecture that the Bernstein theorem is true if the closure of the image under the Gauss map intersects the ideal boundary of hyperbolic space at exactly one point. The most natural geometric candidate whose closure intersects the ideal boundary at exactly one point is the so called "horoball ball" which is defined in the next section. If we use the upper half-plane model for $\mathbb{H}^{m}(-1)$ so that $\mathbb{H}^{m}(-1)$ has coordinate 
$\left(\gamma_{1}, \cdots, \gamma_{m}\right)$ with $\gamma_{m}>0$ and the metric

$$
d s^{2}=\frac{d \gamma_{1}^{2}+\cdots+d \gamma_{m}^{2}}{\gamma_{m}^{2}}
$$

then the set $\left\{\left(\gamma_{1}, \cdots, \gamma_{m}\right) \in \mathbb{H}^{m}(-1): \gamma_{m}>c>0\right\}$ is a horoball for any positive constant $c$. If we use the Poincáre disk model $D$ for the hyperbolic space, then a horoball is a smaller disk that is contained $D$ and intersects $D$ at exactly one boundary point of $D$.

Now, our main theorem can be stated as follows:

Main Theorem. Let $M$ be a complete spacelike constant mean curvature hypersurface in Lorentz-Minkowski space $\mathbb{R}_{1}^{m+1}$. Let $g$ be a function on $M$ such that $e^{-g}$ is superharmonic. If the image of $M$ under the Gauss map $\gamma: M \rightarrow \mathbb{I}^{m}(-1)$ lies in $\left\{\left(\gamma_{1}, \cdots, \gamma_{m}\right): \gamma_{m}>e^{g}\right\}$ then $M$ is necessarily a hyperplane. In particular, this is the case if the Gauss image is contained in the half-space $\left\{\gamma_{m}>c\right\}$ for some constant $c>0$.

Our proof of the Main Theorem is in similar spirit to that of Cheng-Yau [C-Y] and Xin [Xin1]. The key step in the proof is to obtain a gradient estimate for the length of the second fundamental form of the hypersurface. This is done in section 3. The new gradient estimate (Theorem 3.1), which extends an earlier result of Shen [Sh], can be considered as the main analytic result of this paper. It is presented with some general curvature assumptions on both doamin and target manifolds because the result is of interest in its own right for the study of harmonic maps.

Acknowledgement. The second author would like to thank Professor Carolyn Gordon and Professor Richard Schoen for their interest in the result.

\section{Preliminaries}

In this section we shall recall some basic facts and formulas in Lorentzian geometry.

Let $L$ be an $(m+1)$-dimensional Lorentzian manifold with Lorentzian metric $\bar{g}$ of signature $(-,+, \cdots,+)$. Let $\left\{e_{0}, e_{1}, \cdots, e_{m}\right\}$ be a local Lorentzian 
orthonormal moving frame in $L$. Let $\omega_{0}, \omega_{1}, \cdots, \omega_{m}$ be its dual frame so that $\bar{g}=-\omega_{0}^{2}+\sum_{i=1}^{m} \omega_{i}^{2}$. We adopt the convention that Latin indices run from 1 to $m$ and Greek indices run from 0 to $m$. The Lorentzian connection forms $\omega_{\alpha \beta}$ of $L$ are uniquely determined by the structure equations:

$$
\begin{array}{r}
d \omega_{0}=\omega_{0 i} \wedge \omega_{i}, \\
d \omega_{i}=-\omega_{i 0} \wedge \omega_{0}+\omega_{i j} \wedge \omega_{j}, \\
\omega_{\alpha \beta}=-\omega_{\beta \alpha} .
\end{array}
$$

The Covariant derivatives are defined by the following equations

$$
\begin{array}{r}
D e_{0}=\omega_{0 i} e_{i}, \\
D e_{i}=\omega_{i j} e_{j}-\omega_{i 0} e_{0} .
\end{array}
$$

The curvature forms $\bar{\Omega}$ of $L$ are given by

$$
\begin{aligned}
\bar{\Omega}_{0 i}=d \omega_{0 i}-\omega_{0 k} & \wedge \omega_{k i}, \\
\bar{\Omega}_{i j}=d \omega_{i j}+\omega_{i 0} & \wedge \omega_{0 j}-\omega_{i k} \wedge \omega_{k j}, \\
\bar{\Omega}_{\alpha \beta}=-\frac{1}{2} \bar{R}_{\alpha \beta \gamma \delta} \omega_{\gamma} & \wedge \omega_{\delta},
\end{aligned}
$$

where $\bar{R}_{\alpha \beta \gamma \delta}$ are components of the curvature tensor $\bar{R}$ of $L$.

Let $M$ be a spacelike hypersurface in a Lorentzian manifold $L$. We can choose a local Lorentzian orthonormal moving frame $\left\{e_{0}, e_{1}, \cdots, e_{m}\right\}$ in $L$ such that, restricted to $M$, the vectors $e_{1}, \cdots, e_{m}$ are tangential to $M$ and $e_{0}$ is normal to $M$. So the form $\omega_{0}$ is a zero form on the hypersurface $M$ and the induced Riemannian metric $g$ of $M$ is

$$
g=\sum_{i} \omega_{i}^{2} .
$$

Hence the induced structure equations of $M$ are

$$
\begin{array}{r}
d \omega_{i}=\omega_{i k} \wedge \omega_{k}, \\
d \omega_{i j}=\omega_{i k} \wedge \omega_{k j}-\omega_{i 0} \wedge \omega_{0 j}+\bar{\Omega}_{i j}, \\
\omega_{i j}=-\omega_{j i}, \\
\Omega_{i j}=d \omega_{i j}-\omega_{i k} \wedge \omega_{k j}=-\frac{1}{2} R_{i j k l} \omega_{k} \wedge \omega_{l},
\end{array}
$$


where $\Omega_{i j}$ and $R_{i j k l}$ are the curvature forms and the curvature tensors of $M$, respectively.

Since $\omega_{0}=0$ on $M$, equation (4) shows that

$$
\omega_{0 i} \wedge \omega_{i}=0
$$

The Cartan's lemma then implies that

$$
\omega_{i 0}=h_{i j} \omega_{j}
$$

where $h=h_{i j} \omega_{i} \otimes \omega_{j}$ is called the second fundamental form of $M$. We then obtain the Gauss equation

$$
R_{i j k l}=\bar{R}_{i j k l}-\left(h_{i k} h_{j l}-h_{i l} h_{j k}\right) .
$$

By taking the trace, we get the equation for the Ricci tensors

$$
R_{i j}=\bar{R}_{i j}+h_{i k} h_{k j}-m H h_{i j}
$$

where $H=\frac{1}{m} \sum_{i} h_{i i}$ is the mean curvature of $M$ in $L$. If $L$ has Ricci curvature bounded from below by a constant $k_{L}$, then the lower bound of the Ricci curvature of $M$ is given by

$$
\operatorname{Ric}_{M} \geq k_{L}-\frac{1}{4} m^{2} H^{2}
$$

We also like to introduce the notion Busemann function and its level set in order to set up our results. Suppose that $N$ is a complete Riemannian manifold of nonpositive curvature and let $c: \mathbb{R} \rightarrow N$ be a unit speed geodesic. Then we call the union of balls $B_{c}=\bigcup_{t>0} B_{t}(c(t))$ the horoball with center at infinity $c(\infty)$. For $x \in N$, the function $t \rightarrow t-d(x, c(t))$ is bounded from above and monotonically increasing. So we can define the Busemann function by

$$
B(x)=\lim _{t \rightarrow+\infty}(t-d(x, c(t))) .
$$

It is known (see [He-Ho] and [B-Gr-S]) that the Busemann function $B$ is a concave $C^{2}$-function with $|\nabla B|=1$.

We end this section by providing a proof of the well-known result that the Gauss map of a constant mean curvature spacelike hypersurface is a harmonic map into hyperbolic space (see [Mi], [Is] ). The relation between the second 
fundamental form and the derivative of the Gauss map will become clear in the computation.

The Gauss map is a map defined by

$$
f=e_{0}: M \rightarrow H^{m}
$$

We can think of $\left\{e_{i}\right\}$ as an orthonormal basis for $H^{m}$ at the point $e_{0}$. Let $\left\{\theta_{k}\right\}$ be the dual basis for $H^{m}$ and $\left\{\theta_{k l}\right\}$ be its connection form. Then we have

$$
f^{*} \theta_{k}=\omega_{0 k}=h_{i k} \omega_{i}
$$

Hence we see that

$$
f_{i}^{k}=h_{i k}
$$

This implies that

$$
|h|^{2}=|\nabla f|^{2}
$$

By the second structure equation, we have

$$
d \omega_{k 0}=-\omega_{k l} \wedge \omega_{l 0}
$$

So we know that

$$
f^{*} \theta_{i j}=\omega_{i j}
$$

Differentiating (6), we get

$$
\begin{aligned}
& f_{i j}^{k} \omega_{j}+f^{k j} \omega_{j i}-f^{l i} f^{*} \theta_{k l}=d f_{i}^{k} \\
= & d h_{i k}=h_{k i l} \omega_{j}+h_{k j} \omega_{j i}+h_{l i} \omega_{l k} .
\end{aligned}
$$

Hence we obtain that

$$
f_{i j}^{k}=h_{k i j}
$$

Taking the trace, we get

$$
\sum_{i} f_{i i}^{k}=\sum_{i} h_{k i i}=n H_{k}
$$

by the symmetry of the second fundamental form $h$. So $f$ is harmonic if and only if the mean curvature $H$ is constant. 


\section{The Proof of Main Theorem}

In this section, we prove the Main Theorem stated in the introduction. The main analytic tool involved is the following gradient estimate for harmonic maps.

Theorem 3.1. Suppose that $M$ is a complete Riemannian manifold with Ricci curvature Ric $_{M} \geq-k^{2}$ for some $k \geq 0$ and $N$ is a simply-connected complete Riemannian manifold with sectional curvature $K_{N} \leq-1$. If $f$ : $M \rightarrow N$ is a harmonic map and $B$ is a Busemann function on $N$ such that $B \circ f \geq g$ for some function $g$ satisfying $\triangle e^{-g} \leq 0\left(^{*}\right)$, then we have

$$
\sup _{B_{\frac{a}{2}}\left(x_{0}\right)} \frac{|\nabla f|}{B \circ f-g} \leq c(m, n) \frac{1+k a}{a}
$$

where $a>0$ is an arbitrary constant.

From Theorem 3.1, one can also obtain the following estimate which can be regarded as an extension of Yau's Liouville Theorem for harmonic functions.

Theorem 3.2. Let $f$ be a harmonic function on a complete Riemannian manifold $M$ with Ricci curvature Ric $_{M} \geq-k^{2}$ for some constant $k \geq 0$. If there exists a function $g$ such that $e^{-g} \leq 0$ and $f \geq g$, then

$$
\sup _{B_{\frac{a}{2}}\left(x_{0}\right)} \frac{|\nabla f|}{f-g} \leq c(m) \frac{1+k a}{a} .
$$

for all positive constant $a>0$. In particular, if the Ricci curvature of $M$ is nonnegative, then the harmonic function has to be constant.

Proof of Theroem 3.1. Let $M$ be a complete Riemannian manifold with Ricci curvature $\operatorname{Ric}_{M} \geq-k^{2}$ for some $k \geq 0$ and $N$ be a simply-connected complete Riemannian manifold with sectional curvature $K_{N} \leq-1$. If $f$ : $M \rightarrow N$ is a harmonic map and $B$ is a Busemann function on $N$ such that $B \circ f \geq g$ for some function $g$ satisfying $\triangle e^{-g} \leq 0$. Let

$$
\phi=\frac{|\nabla f|}{B \circ f-g}
$$


then we have

$$
\nabla \phi=\frac{\nabla|\nabla f|}{B \circ f-g}-\frac{|\nabla f|(\nabla B \circ f-\nabla g)}{(B \circ f-g)^{2}}
$$

and

$$
\triangle \phi=\frac{\triangle|\nabla f|}{B \circ f-g}-2 \frac{\nabla(B \circ f-g) \cdot \nabla \phi}{B \circ f-g}-\frac{\phi \triangle B \circ f-\phi \triangle g}{B \circ f-g} .
$$

For harmonic map $f$, we have the following Bochner formula

$$
\frac{1}{2} \triangle|\nabla f|^{2} \geq\left|f_{i j}^{k}\right|^{2}-k^{2}|\nabla f|^{2}
$$

It is known that (see [Sc-Yaul)

$$
\left|f_{i j}^{k}\right|^{2} \geq\left.\left(1+\frac{1}{2 m n}\right)|\nabla| \nabla f\right|^{2},
$$

where $m=\operatorname{dim}(M)$ and $n=\operatorname{dim}(N)$. Combining (10) and (11), we obtain

$$
|\nabla f| \triangle|\nabla f| \geq\left.\epsilon|\nabla| \nabla f\right|^{2}-k^{2}|\nabla f|^{2},
$$

where $\epsilon=\frac{1}{2 m n}$.

Inequalities (12) and (9) imply

$$
\begin{array}{r}
\triangle \phi \geq \epsilon \frac{\left.|\nabla| \nabla f\right|^{2}}{|\nabla f|(B \circ f-g)}-k^{2} \phi \\
-2 \frac{(\nabla B \circ f-\nabla g) \cdot \nabla \phi}{B \circ f-g}-\frac{\phi \triangle(B \circ f-g)}{B \circ f-g} .
\end{array}
$$

We know that

$$
\begin{aligned}
& -2 \frac{(\nabla B \circ f-\nabla g) \cdot \nabla \phi}{B \circ f-g} \\
= & -(2-2 \epsilon) \frac{(\nabla B \circ f-\nabla g) \cdot \nabla \phi}{B \circ f-g}-2 \epsilon \frac{(\nabla B \circ f-\nabla g) \cdot \nabla \phi)}{B \circ f-g} \\
= & -(2-2 \epsilon) \frac{(\nabla B \circ f-\nabla g) \cdot \nabla \phi}{B \circ f-g}-2 \epsilon \frac{(\nabla B \circ f-\nabla g) \cdot \nabla|\nabla f|}{(B \circ f-g)^{2}}
\end{aligned}
$$




$$
\begin{aligned}
& +2 \epsilon \frac{|\nabla B \circ f-\nabla g|^{2}|\nabla f|}{(B \circ f-g)^{3}} \\
& \geq-(2-2 \epsilon) \frac{(\nabla B \circ f-\nabla g) \cdot \nabla \phi}{B \circ f-g}-2 \epsilon \frac{|\nabla B \circ f-\nabla g||\nabla| \nabla f||}{(B \circ f-g)^{2}} \\
& +2 \epsilon \frac{|\nabla B \circ f-\nabla g|^{2}|\nabla f|}{(B \circ f-g)^{3}},
\end{aligned}
$$

and

$$
\begin{aligned}
& -2 \epsilon \frac{|\nabla B \circ f-\nabla g||\nabla| \nabla f||}{(B \circ f-g)^{2}} \\
\geq & -\epsilon \frac{|\nabla| \nabla f||^{2}}{(B \circ f-g)|\nabla f|}+\epsilon \frac{|\nabla B \circ f-\nabla g|^{2}|\nabla f|}{(B \circ f-g)^{3}} .
\end{aligned}
$$

Inequalities (13), (14) and (15) then imply that

$$
\begin{array}{r}
\triangle \phi \geq-(2-2 \epsilon) \frac{\nabla(B \circ f-g) \cdot \nabla \phi}{B \circ f-g}+ \\
\epsilon \frac{|\nabla f||\nabla B \circ f-\nabla g|^{2}}{(B \circ f-g)^{3}}-k^{2} \phi-\frac{\phi \triangle B \circ f-\phi \triangle g}{B \circ f-g} .
\end{array}
$$

For any fixed point $x_{0} \in M$, we can define a function $F$ on the ball $B_{a}\left(x_{0}\right)$ by

$$
F(x)=\left(a^{2}-r^{2}\right) \phi(x),
$$

where $r(x)=\operatorname{dist}\left(x, x_{0}\right)$ is the distance function on $M$. Without lossing of generality, we may assume $r \in C^{2}(M)$. If $\nabla f$ is not identically zero, then $F$ must attain its maximum at some interior point $x^{*}$. By the mzximum principle, we have

$$
\begin{aligned}
& \nabla F\left(x^{*}\right)=0, \\
& \triangle F\left(x^{*}\right) \leq 0 .
\end{aligned}
$$

By (17) and (18), we have at $x^{*}$

$$
\frac{\nabla r^{2}}{a^{2}-r^{2}}=\frac{\nabla \phi}{\phi},
$$


and

$$
-\frac{\triangle r^{2}}{a^{2}-r^{2}}+\frac{\triangle \phi}{\phi}-\frac{2 \nabla r^{2} \cdot \nabla \phi}{\left(a^{2}-r^{2}\right) \phi} \leq 0 .
$$

It follows that

$$
\frac{\triangle \phi}{\phi}-\frac{\triangle r^{2}}{a^{2}-r^{2}}-\frac{2\left|\nabla r^{2}\right|^{2}}{\left(a^{2}-r^{2}\right)^{2}} \leq 0
$$

Using the Laplacian comparison theorem (see Gr-Wu ), we obtain

$$
\triangle r^{2} \leq C(1+k r)
$$

Also, it is well-known that

$$
|\nabla r|=1
$$

Using inequality (16), we obtain

$$
\epsilon \frac{|\nabla B \circ f-\nabla g|^{2}}{(B \circ f-g)^{2}}-\frac{\triangle(B \circ f-g)}{B \circ f-g}-\frac{4(1-\epsilon) r}{a^{2}-r^{2}} \frac{\nabla(B \circ f-g) \cdot \nabla r}{B \circ f-g} \leq A,
$$

where

$$
A=k^{2}+\frac{C(1+k r)\left(a^{2}-r^{2}\right)+8 r^{2}}{\left(a^{2}-r^{2}\right)^{2}} .
$$

Using $a b \geq-\frac{\epsilon}{2} a^{2}-\frac{2}{\epsilon} b^{2}$ and $|\nabla r|=1$, (19) can be simplified as

$$
\frac{\epsilon}{2} \frac{|\nabla B \circ f-\nabla g|^{2}}{(B \circ f-g)^{2}}-\frac{\triangle(B \circ f-g)}{B \circ f-g} \leq A^{\prime}
$$

where

$$
A^{\prime}=k^{2}+C \frac{(1+k r)\left(a^{2}-r^{2}\right)+r^{2}}{\left(a^{2}-r^{2}\right)^{2}} .
$$

It is easy to see that

$$
|\nabla B \circ f-\nabla g|^{2} \geq \frac{1}{2}|\nabla B \circ f|^{2}-|\nabla g|^{2} .
$$

Since $B$ is a horofunction, we can always assume that $B \circ f \geq 1+g$. Combining (20) and (21), we have

$$
\frac{\epsilon}{4} \frac{|\nabla B \circ f|^{2}}{(B \circ f-g)^{2}}-\frac{\triangle B \circ f}{B \circ f-g}+\frac{\triangle g}{B \circ f-g}-\frac{\epsilon}{2} \frac{|\nabla g|^{2}}{(B \circ f-g)^{2}}-A^{\prime} \leq 0 .
$$


By Condition $(*)$, we see that

$$
|\nabla g|^{2}-\triangle g \leq 0
$$

So

$$
\frac{\triangle g}{B \circ f-g}-\frac{\epsilon}{2} \frac{|\nabla g|^{2}}{(B \circ f-g)^{2}} \geq 0
$$

From (22) and (23), we get

$$
\frac{\epsilon}{4} \frac{|\nabla B \circ f|^{2}}{(B \circ f-g)^{2}}-\frac{\triangle B \circ f}{B \circ f-g} \leq A^{\prime} .
$$

If we choose a local polar coordinate chart around $p=f(x)$ such that $e_{1}=$ $\nabla B$, the radial direction. Then we have

$$
|\nabla B \circ f|^{2}=\sum_{i=1}^{m}\left|f_{i}^{\alpha} B_{\alpha}(f)\right|^{2} \geq \sum_{i=1}^{m}\left|f_{i}^{1}(x)\right|^{2} .
$$

Applying the Hessian comparison theorem, we have

$$
B_{\alpha \beta}=-\lim _{t \rightarrow \infty} d_{\alpha \beta}\left(x, c(t) \leq-\lim _{t \rightarrow \infty} \operatorname{coth}\left(d(x, c(t)) H_{\alpha \beta}(p) \leq-H_{\alpha \beta}(p),\right.\right.
$$

where $H_{\alpha \beta}=\gamma_{\alpha \beta}-d B_{\alpha} d B_{\beta}$ and $\gamma_{\alpha \beta}$ is the metric on $N$.

Since $f$ is harmonic,

$$
\triangle B \circ f(x)=g^{i j} f_{i}^{\alpha} f_{j}^{\beta} B_{\alpha \beta} \leq-\sum_{\alpha=2}^{n} \sum_{i=1}^{m}\left|f_{i}^{\alpha}\right|^{2} .
$$

Combining (25), (26) and using the assumption that $B \circ f-g \geq 1$, we obtain the the following key inequality

$$
\frac{\epsilon}{4} \frac{|\nabla B \circ f|^{2}}{(B \circ f-g)^{2}}-\frac{\triangle B \circ f}{B \circ f-g} \geq \frac{\epsilon}{4} \frac{|\nabla f|^{2}}{(B \circ f-g)^{2}} .
$$

Inequalities (24) and (27) then imply that

$$
\frac{\epsilon}{4} \frac{|\nabla f|^{2}}{(B \circ f-g)^{2}} \leq A^{\prime} .
$$


which is equivalent to

$$
\epsilon F^{2} \leq A^{\prime}
$$

Now the gradient estimate (8) follows easily from (28). This completes the proof of Theorem 3.1.

Using Theorem 3.1, we now present the proof of the Main Theorem.

Proof of Main Theorem. We first use the generalized maximum principle due to Omori $\mathrm{Om}$ and Yau Yau to prove that the constant mean curvature is actually zero.

Let $f$ be the Gauss map from $M$ to the hyperbolic space $\mathbb{H}^{m}$ and $B$ be a Busemann function on $\mathbb{H}^{m}$. Define the function $h=-(B \circ f-g)$, where $e^{-g}$ is superharmonic on $M$. Then it is easy to see that $h \in C^{2}(M)$ He-Ho and $h$ is bounded from above. Since the Ricci curvature of $M$ is bounded from below by a constant, we have the Omori-Yau Om, Yau maximum principle for complete manifold. Therefore, for $\forall \epsilon>0$, and $\forall x_{0} \in M$, there exists a point $x \in M$ such that

$$
\begin{aligned}
& h(x) \geq h\left(x_{0}\right), \\
& |\nabla h|\left(x_{0}\right)<\epsilon,
\end{aligned}
$$

$$
(\triangle h)<\epsilon
$$

From our estimates (25) and (26) in the proof of Theorem 3.1 and our Condition $(*)$, we know that

$$
\begin{array}{r}
|\nabla h|^{2}+\triangle h=|\nabla B \circ f|^{2}+|\nabla g|^{2}-2 \nabla B \circ f \nabla g-\triangle B \circ f+\triangle g \\
\geq\left(\frac{1}{2}|\nabla B \circ f|^{2}-\triangle B \circ f\right)+\left(\triangle g-|\nabla g|^{2}\right) \\
\geq \frac{1}{2}|\nabla f|^{2}=\frac{1}{2} \sum_{i, j} h_{i j}^{2} \geq \frac{m}{2} H^{2}
\end{array}
$$

Since $H$ is a constant, inequalities (29), (30) and (31) force $H \equiv 0$. Now it follows from (5) that the Ricci curvature of $M$ is nonnegative. Hence our 
gradient estimate (8) in Theorem 3.1, with $k=0$, implies

$$
\sup _{B_{\frac{a}{2}}\left(x_{0}\right)} \frac{|\nabla f|}{B \circ f-g} \leq c(m, n) \frac{1}{a} .
$$

So $|\nabla f|^{2}=0$ by letting $a \rightarrow \infty$. Hence (7) implies that the second fundamental form of $M$ is identically zero. Therefore $M$ must be a hyperplane. This completes the proof of our Main Theorem.

The proof of Theorem 3.2 is exactly the same as the proof of Theorem 3.1. For $N=\mathbb{H}^{n}$ and any fixed constant $c>0$, if we use the coordinate chart $z=\left(z_{1}, \cdots, z_{n}\right)$ with $z_{n}>0$ and choose

$$
\gamma=\left\{\left(0, \cdots, 0, z_{n}\right): z_{n} \geq c\right\}
$$

to be a geodesic ray. Then the Busemann function for $\gamma$ is

$$
B_{c}(z)=\ln \left(\frac{z_{n}}{c}\right)
$$

For harmonic function $z=f(x)$, we can let

$$
y=\ln z .
$$

Then

$$
d z^{2}=\frac{d y^{2}}{y^{2}} .
$$

Analytically, we remark that in order to obtain an upper bound for $\frac{|\nabla f|}{\phi \circ f-\tilde{g}}$, it suffices to have the followings:

$$
|\nabla \phi \circ f|^{2}-\triangle \phi \circ f \geq c|\nabla f|^{2}
$$

and

$$
|\nabla \tilde{g}|^{2}-\triangle \tilde{g} \leq 0
$$

Inequality (27) is just a consequence of (32) and (33) since we can always make $\phi \circ f-g \geq 1$.

Clearly, our assumptions in Theorem 3.2 imply both (32) and (33). Therefore, Theorem 3 is true. 


\section{Applications}

In this section we are going to apply our Main Theorem to study the Bernstein problem on spacelike submanifolds with parallel mean curvature vector in semi-Euclidean space with higher codimensions. No much is known in this direction. Our work is inspired by Xin's original paper Xin2 which proves a nice splitting theorem for certain spacelike surface in $\mathbb{R}_{2}^{4}$.

Let $\mathbb{R}_{n}^{m+n}$ be a $m+n$-dimensional semi-Euclidean space with the semiRiemannian metric

$$
d s^{2}=d x_{1}^{2}+\cdots+d x_{m}^{2}-d x_{m+1}^{2}-\cdots-d x_{m+n}^{2} .
$$

Let $\mathrm{M}$ be a spacelike oriented $m$-dimensional submanifold of $\mathbb{R}_{n}^{m+n}$. Following [Xin2], one can define the generalized Gauss map $\gamma: M \rightarrow G_{m, n}^{m}$, where $G_{m, n}^{m}$ is a Cartan-Hardamard manifold formed by replacing complex numbers with real numbers in the bounded symmetric domain of first type $G_{m, n}^{m}(C)$ (see Xin2]). In the following we are going to study spacelike surfaces with parallel mean curvature vector in $\mathbb{R}_{2}^{4}$, a 4-manifold with signature $(+,+,-,-)$.

Lemma 4.1.([He] $) G_{2,2}^{2}$ is isometric to $\mathbb{H}^{2}(-1) \times \mathbb{H}^{2}(-1)$.

In the following we will identify $G_{2,2}^{2}$ with $\mathbb{H}^{2}(-1) \times \mathbb{H}^{2}(-1)$. Then for any given map $f: M \rightarrow G_{2,2}^{2}$, we can write $f=\left(f_{1}, f_{2}\right)$, where $f_{i}=\pi_{i} \cdot f$ and $\pi_{i}: G_{2,2}^{2} \rightarrow \mathbb{H}^{2}(-1)$ is the pojection of $G_{2,2}^{2}$ into its $i$-th factor, $i=1,2$.

Now, we can state a Bernstein theorem for spacelike surface with parallel mean curvature vector in $\mathbb{R}_{2}^{4}$.

Theorem 4.2. Let $M$ be a complete spacelike surface with parallel mean curvature vector in $\mathbb{R}_{2}^{4}$ and let $\gamma=\left(\gamma_{1}, \gamma_{2}\right): M \rightarrow G_{2,2}^{2}$ be the Gauss map. If the image under the map $\gamma_{1}$ (or $\gamma_{2}$ ) lies in a horoball in $\mathbb{H}^{2}(-1)$, then $M$ must be a plane.

We choose a local Lorentzian orthonormal frame $\left\{e_{1}, e_{2}, e_{3}, e_{4}\right\}$ along $M$ such that $e_{1}, e_{2}$ are tangent to $M$ with dual frame $\left\{\omega_{1}, \omega_{2}, \omega_{3}, \omega_{4}\right\}$. In the following, we adopt the convention that the Latin indices run from 1 to 2 and the Greek indices run from 3 to 4 . The induced Riemannian metric of 
$M$ is

$$
g=\omega_{1}^{2}+\omega_{2}^{2}
$$

So the structure equations of $M$ are

$$
\begin{array}{r}
d \omega_{i}=\omega_{i k} \wedge \omega_{k}, \\
\omega_{i k}=-\omega_{k i}, \\
d \omega_{i j}=\omega_{i k} \wedge \omega_{k j}-\omega_{i \alpha} \wedge \omega_{\alpha j}, \\
\Omega_{i j}=d \omega_{i j}-\omega_{i k} \wedge \omega_{k j}=-\frac{1}{2} R_{i j k l} \omega_{k} \wedge \omega_{l} .
\end{array}
$$

By Cartan's lemman, we have

$$
\omega_{i \alpha}=h_{i j}^{\alpha} \omega_{j},
$$

where $h_{i j}^{\alpha}$ are components of the second fundamental form of $M$ in $\mathbb{R}_{2}^{4}$. The mean curvature vector of $M$ in $\mathbb{R}_{2}^{4}$ is

$$
\vec{H}=\frac{1}{2} g^{i j} h_{i j}^{\alpha} e_{\alpha}
$$

We then obtain from the Gauss equation that the sectional curvature of $M$ is

$$
R_{i j k l}=-\left(h_{i k}^{\alpha} h_{j l}^{\alpha}-h_{i l}^{\alpha} h_{j k}^{\alpha}\right),
$$

and the Ricci curvature of $M$ is

$$
R_{j l}=g^{i k} R_{i j k l}=-\left(h_{i i}^{\alpha} h_{j l}^{\alpha}-h_{i l}^{\alpha} h_{j i}^{\alpha}\right) .
$$

The second structure equations on the induced normal connection of $M$ in $\mathbb{R}_{2}^{4}$ are

$$
\begin{aligned}
d \omega_{\alpha \beta} & =-\omega_{\alpha \gamma} \wedge \omega_{\gamma \beta}+\Omega_{\alpha \beta}, \\
\Omega_{\alpha \beta} & =-\frac{1}{2} R_{\alpha \beta \gamma \delta} \omega_{\gamma} \wedge \omega_{\delta}, \\
R_{\alpha \beta i j} & =-\left(h_{k i}^{\alpha} h_{k j}^{\beta}-h_{k j}^{\alpha} h_{k i}^{\beta} .\right)
\end{aligned}
$$

Define the covariant derivative of $h_{i j}^{\alpha}$ by

$$
h_{i j k}^{\alpha} \omega_{k}=d h_{i j}^{\alpha}+h_{k j}^{\alpha} \omega_{k i}+h_{i k}^{\alpha} \omega_{k j}-h_{i j}^{\beta} \omega \alpha \beta .
$$


It is easy to see that $h_{i j k}^{\alpha}$ is symmetric in $i, j, k$.

We say that $M$ has parallel mean curvature vector if

$$
D \vec{H}=\frac{1}{2} g^{i j} h_{i j k}^{\alpha} \omega_{k} e_{\alpha}=0
$$

holds everywhere in $M$.

For the Gauss map

$$
\gamma: M \rightarrow G_{2,2}^{2},
$$

so we have

$$
\gamma^{*} \omega_{\alpha i}=h_{i j}^{\alpha} \omega_{j} .
$$

It follows from Equations (34) and (35) that the Gauss map $\gamma$ is harmonic if and only if $M$ has parallel mean curvature vector.

It is computed in Xin2 that the canonical metric on $G_{2,2}^{2}=\mathbb{H}^{2}(-1) \times$ $\mathbb{I H}^{2}(-1)$ is

$$
d s_{1}^{2}+d s_{2}^{2},
$$

where

$$
d s_{1}^{2}=\frac{1}{2}\left[\left(\omega_{13}+\omega_{24}\right)^{2}+\left(\omega_{23}-\omega_{14}\right)^{2}\right]
$$

is the induced metric of the first factor.

Equations (35) and (36) then imply that

$$
\begin{aligned}
& \gamma_{1}^{*}\left(\omega_{13}+\omega_{24}\right)=\left(h_{11}^{3}+h_{12}^{4}\right) \omega_{1}+\left(h_{12}^{3}+h_{22}^{4}\right) \omega_{2}, \\
& \gamma_{1}^{*}\left(\omega_{23}-\omega_{14}\right)=\left(h_{11}^{4}-h_{12}^{3}\right) \omega_{1}+\left(h_{12}^{4}-h_{22}^{3}\right) \omega_{2} .
\end{aligned}
$$

If we write

$$
\begin{aligned}
& \gamma_{1}^{*}\left(\omega_{13}+\omega_{24}\right)=a_{11} \omega_{1}+a_{12} \omega_{2}, \\
& \gamma_{1}^{*}\left(\omega_{23}-\omega_{14}\right)=a_{21} \omega_{1}+a_{22} \omega_{2},
\end{aligned}
$$

then we have

$$
\begin{aligned}
& a_{11}=h_{11}^{3}+h_{12}^{4}, \\
& a_{12}=h_{12}^{3}+h_{22}^{4}, \\
& a_{21}=h_{11}^{4}-h_{12}^{3}, \\
& a_{22}=h_{12}^{4}-h_{22}^{3} .
\end{aligned}
$$


Consequently,

$$
\begin{aligned}
& a_{111}=h_{111}^{3}+h_{121}^{4}, \\
& a_{112}=h_{112}^{3}+h_{122}^{4}, \\
& a_{121}=h_{121}^{3}+h_{221}^{4}, \\
& a_{122}=h_{122}^{3}+h_{222}^{4}, \\
& a_{211}=h_{111}^{4}-h_{121}^{3}, \\
& a_{212}=h_{112}^{4}-h_{122}^{3}, \\
& a_{221}=h_{121}^{4}-h_{221}^{3}, \\
& a_{222}=h_{122}^{4}-h_{222}^{3} .
\end{aligned}
$$

Thus, the map $\gamma_{1}$ is harmonic if and only if

$$
\begin{gathered}
h_{111}^{3}+h_{122}^{3}+h_{211}^{4}+h_{222}^{4}=0, \\
h_{111}^{4}+h_{122}^{4}-h_{211}^{3}-h_{222}^{3}=0 .
\end{gathered}
$$

Similarly, the map $\gamma_{2}$ is harmonic if and only if

$$
\begin{gathered}
h_{111}^{3}+h_{122}^{3}-h_{211}^{4}-h_{222}^{4}=0 \\
h_{111}^{4}+h_{122}^{4}+h_{211}^{3}+h_{222}^{3}=0 .
\end{gathered}
$$

If $M$ has parallel mean curvature vector, then

$$
D \vec{H}=\overrightarrow{0}
$$

So $M$ has parallel mean curvature vector if and only if

$$
\begin{aligned}
& h_{111}^{3}+h_{122}^{3}=0, \\
& h_{211}^{3}+h_{222}^{3}=0, \\
& h_{111}^{4}+h_{122}^{4}=0, \\
& h_{211}^{4}+h_{222}^{4}=0,
\end{aligned}
$$

Therefore, it is easy to see from the Equations (37), (38) and (39) that a spacelike surface in $\mathbb{R}_{2}^{4}$ has parallel mean curvature vector if and only if both $\gamma_{1}$ and $\gamma_{2}$ are harmonic maps. 
In the following, we assume that $M$ has parallel mean curvature vector and we specify our local orthonormal frame $\left\{e_{1}, e_{2}, e_{3}, e_{4}\right\}$ so that

$$
e_{3}=\frac{\vec{H}}{|\vec{H}|} .
$$

Since $e_{3}$ is parallel in the normal bundle, we know that

$$
\omega_{34}=0,
$$

and $h_{i j}^{\alpha}$ can be diagonalized simultaneously. Therefore, we have

$$
\begin{gathered}
a_{11}=h_{11}^{3}, \\
a_{12}=h_{22}^{4}, \\
a_{21}=h_{11}^{4}, \\
a_{22}=-h_{22}^{3} .
\end{gathered}
$$

Thus, the energy density of $\gamma_{1}$ is

$$
e\left(\gamma_{1}\right)=\frac{1}{2} \sum_{i j} a_{i j}^{2}=\frac{1}{2}\left[\left(h_{11}^{3}\right)^{2}+\left(h_{22}^{3}\right)^{2}+\left(h_{11}^{4}\right)^{2}+\left(h_{22}^{4}\right)^{2}\right] .
$$

Similarly, we have

$$
e\left(\gamma_{2}\right)=\frac{1}{2}\left[\left(h_{11}^{3}\right)^{2}+\left(h_{22}^{3}\right)^{2}+\left(h_{11}^{4}\right)^{2}+\left(h_{22}^{4}\right)^{2}\right]=e\left(\gamma_{1}\right) .
$$

If the image under the harmonic map $\gamma_{1}$ lies in some horoball in $\mathbb{H}^{2}(-1)$, then we can apply our gradient estimates in section 3 to show that

$$
e\left(\gamma_{1}\right)=0,
$$

and,

$$
e(\gamma)=e\left(\gamma_{1}\right)+e\left(\gamma_{2}\right)=2 e\left(\gamma_{1}\right)=0 .
$$

Therefore, $\gamma$ is a constant map and consequently $M$ must be a plane. This completes our proof for Theorem 4.2. 


\section{References}

[A] F.J. Almgren, Jr., Some interior regularity theorems for minimal surfaces and an extension of Bernstein's tehorem, Ann. of Math., 84 (1966), 277-292.

[B] S. Bernstein, Sur un théorème de aux dérivées partielles du type elliptique, Comm. de la Soc. Math. de Kharkov (2é sér.) 15 (1915-1917), $38-45$.

[BDG] E. Bombieri, E. De Giorgi, E. Giusti, Minimal cones and the Bernstein problem, Invent. Math. 7 (1969), 243-268.

[B-Gr-S] W. Ballman, M. Gromov, V. Schroeder, Manifolds of nonpositive curvature, Progr. Math., vol. 66, Birkhauser, Boston, 1985.

[Ca] E. Calabi, Examples of Bernstein problems for some nonlinear equations, Proc. Sympos. Pure Appl. Math. 15 (1968), 223-230.

[Ch] S.S. Chern, On the curvatures on a piece of hypersurface in Euclidean space, Abh. Math. Sem. Hamburg, 29, 1964.

[Cho- $\operatorname{Tr}]$ H. Choi, A. Treibergs, Gauss maps on spacelike constant mean curvature hypersurfaces of Minkowski space, J. Diff. Geom. 32 (1990), $775-817$.

[C-Y] S.Y. Cheng, S.T. Yau, Maximal hypersurfaces in the LorentzMinkowski spaces, Ann. of Math 104 (1976), 407-419.

[DG] E. De Giorgi, Una estensione del teorema di Berstein, Ann. Scuola Norm. Sup. Pisa 19 (1965), 79-85.

[E-L] J. Eells, L. Lemaire, A report on harmonic mpas, Bull London Math. Soc. 10 (1978), 1-68.

[Gr-Wu] R. Greene, H. Wu, Function theory on manifolds which possess a pole, Lecture Notes in Math., vol. 699, Springer-Verlag, New York, 1979.

[F] W. Fleming, On the oriented Plateau problem, Rend. Circ. Mat. Palerino 11 (1962), 69-90. 
[H-O-S] D. Hoffman, R. Osserman, R. Schoen, On the Gauss map of complete surfaces of constant mean curvature in $R^{3}$ and $R^{4}$, Comment. Math. Helv., 57 (1982), 519-531.

[Hel] S. Helgason, Differential geometry, Lie groups and symmetric spaces, Academic Press, 1978.

[He-Ho] E. Heintze, H. Im Hof, Geometry of horospheres, J. Diff. Geom. 12 (1977), 481-490.

[Ish] T. Ishihara, The harmonic Gauss maps in a generalized sense, J London Math. Soc. 26 (1982) 104-112.

[Mi] T. K. Milnor, Harmonic maps and classical surface theory in Minkowski space, trans. Amer. Math. Soc. 280 (1983) 161-185.

[Om] H. Omori, Isometric immersions of Riemannian manifolds, J. Math. Soc. Japan 19 (1967), 205-241.

[Pa] B. Palmer, The Gauss map of a spacelike constant mean curvature hypersurface of Minkowski space, Comment. Math. Helvetici 65 (1990), $52-57$.

[R-V] E. Ruh, J. Vilms, The tension field of the Gauss map, Trans. Amer. Math. Soc. 149 (1970), 569-573.

[Sc-Yau] R. Schoen, S.T. Yau, Harmonic maps and the topology of stable hypersurfaces and manifolds with non-negative Ricci curvature, Comment. Math. Helvetici 51 (1976), 333-341.

[Sh] Y. Shen, A Liouville theorem for harmonic maps, Amer. J. Math., 117(1995), 773-785.

[SJ] J. Simons, Minimal varieties in Riemannian manifolds, Ann. of Math. 88 (1968), 62-105.

[Su] H. Sun, The first eigen-value of the totally real submanifolds with constant mean curvature in the complex space form, Memo. Fac. of Sci., Kyushu Univ., Ser. A (1993), 411-419. 
[Tr] A.E. Treibergs, Entire spacelike hypersurfaces of constant mean curvature in Minkowski space, Invent. Math., 66 (1982), 39-56.

[Xin1] Y.L. Xin, On the Gauss image of a spacelike hypersurface with constant mean curvature in Minkowski space, Comment. Math. Helvetici 66 (1991), 590-598.

[Xin2] Y.L. Xin, Harmonic maps of bounded symmetric domains, Math. Ann. 303(1995), 417-433.

[Yau] S.T. Yau, Harmonic functions on complete Riemannian manifolds, Comm. Pure Appl. Math. 28(1975), 201-228.

Department of Mathematics, Texas A\&M University, College Station, TX 77843

E-mail address: cao@math.tamu.edu

Department of Mathematics, Dartmouth College, Hanover, NH 03755

E-mail address: ying.shen@dartmouth.edu

Department of Mathematics, Dartmouth College, Hanover, NH 03755

E-mail address: shunhui.zhu@dartmouth.edu 